\title{
DETERMINATION OF SUBJECTS' SIGNIFICANCE RATE AND OPTIMAL INFORMATION CONTROL IN SOCIAL NETWORKS
}

\author{
Sharif Guseynov \\ Liepaja University, Latvia \\ Aleksandrs Bereznojs \\ ISMA University of Applied Sciences, Latvia \\ Ventspils University of Applied Sciences, Latvia \\ Jekaterina Aleksejeva \\ Liepaja University, Latvia \\ Riga Secondary School 34, Latvia
}

\begin{abstract}
This paper examines social networks, where each agent is characterized by some dynamic parameters, the dynamics of which is resulting from the influence of other agents having their own objective functions and limiting factors, as well as from control/governing body with its own objective function. In this paper, referring to the type of social networks described above, the following two interrelated problems are investigated: the problem of determining the degree of information influence on social networks; the problem of finding optimal control in social networks.
\end{abstract}

Keywords: influences degree; Nash Equilibrium; optimal control; social networks.

\section{Introduction}

Contemporary social networks represent a social structure consisting of many subjects (i.e. individual and/or collective agents - individuals; households; families; professional groups; organizations; etc.) with a given set of relations (i.e. a set of connections between subjects - dating; friendship; partnership, professional cooperation; common ideological or religious beliefs; political or economic views and opinions; etc.). Referring to the type of considered social network, its subject can be either passive (in the case when the main characteristic of this subject changes according to some given law, based on the characteristics of the subjects related to it) or active (in the case when this subject himself/herself chooses a characteristic, for example, action or inaction, etc.). Mathematical modelling of social networks, as a rule, is limited to consideration of social networks with passive subjects; in some rare cases, networks with active subjects are considered. It is important that when 
modelling real social networks, the main common factors are taken into account, which, in our opinion, are: (a) structural and topological laws and properties of social networks; (b) proper opinions of the subjects of social networks, which can be changed both under the influence of other subjects (fully or partially biased or impartial), as well as due to various off-network factors of the surrounding reality; (c) a degree of authority/significance/trust of the opinions of some subjects for the other subjects; (d) dynamic degree of socio-psychopolitical-economic susceptibility of subjects to influence; (e) the threshold of subjects' sensitivity to the dynamics of the opinions of both other subjects of social networks as well as subjects not being a part of the network; (f) temporary avalanche effects called cascades; (g) the degree of incomplete and/or asymmetric awareness of subjects when they make decisions both in conditions of uncertainty and in cascades; (h) the possibility of forming coalitions; (i) information control. The results of social networks modelling without taking into account at least the above basic general factors cannot claim to be adequate (for instance, see Gubanov, Novikov, \& Chkhartishvili, 2010).

In the late 90s of the last century, there was a massive collapse of the socalled dot-com (.com) companies and this phenomenon, sensitive for the Internet technology industry, has contributed immensely to the revision of the basic laws of social networks, which were considered almost immutable: asymptotic laws about the value of social networks - Sarnoff's, Metcalfe's, and Reed's Laws (for instance, see Kovarik, 2015; Simeonov, 2006; Reed, 1999, 2001; Farris, Pfeifer, \& Johnson, 2009); information field formation laws (for instance, see Bukharin, Kovalev, \& Malkov, 2009; Bukharin \& Malkov, 2010; Chernets, Bazlova, \& Ivanova, 2010; Gubanov, 2020; Malkov, 2016; Gubanov et al., 2010); laws of formation, dynamics and influence of opinions (Nguyen, Xiao, Xu, Wu, \& Xia, 2020; Galam, 2012; Xiong \& Liu, 2014; Gubanov, Novikov, \& Chkhartishvili, 2009; Grabich \& Rusinowska, 2010; Rusinowska \& Swart, 2007; see also relatively old work Hoede \& Bakker, 1982, which is important in terms of its subsequent influences); etc. As a result, there have appeared other laws and regulations, methods and approaches, and even qualitatively new concepts (for more information on these methods and concepts see fundamental monographs Jackson, 2008; Gubanov et al., 2010; Fowler \& Christakis, 2011; Amati, Lomi, \& Mira, 2018; Razis, Anagnostopoulos, \& Zeadally, 2020; Johnson, Turnbull, Maher, \& Reisslein, 2021; see also the voluminous article Gubanov et al., 2009). Due to the aforementioned revision, mathematical models of various levels have been constructed and studied (many of them only partially) to describe and understand various aspects of sustainable existence, functioning, safety and reliability, development, value, influence, danger and regulation of social networks. The scientific methods used in this case cover various areas of mathematics: theory of sets, probability theory and 
mathematical statistics, theory of graphs, mathematical logic theory (both Boolean algebra, multivalued logic and temporal logic), discrete optimization, and even linear and non-linear differential and integral equations.

In this paper, we study social networks whose subjects are of a combined nature, i.e. can behave both actively and passively. In other words, we study a social network, each subject of which is characterized by some dynamic parameters, the dynamics of which is determined by the influence of both other subjects and the governing body (in the sense of a stimulation), which has its proper goal (i.e. the stimulating objective function). In addition, it is assumed that each subject also has its proper goal (i.e. the individual objective function) and can choose its action within the limits of the imposed restrictions. In the assumptions made, the essence of the studied problem, i.e. the problem of determining the weights of subjects and finding the optimal information control of subjects in social networks of the selected type is as follows:

- development of an algorithm for determining the subjects' significance coefficients on the basis of the values of a certain set of measured parameters;

- establishing relationships between the subjects' significance coefficients, actions of subjects, types of subjects' activity (i.e., construction of subjects' individual objective functions and its related restrictions);

- finding a necessary and sufficient condition under which the objectives of the subjects reach its maximum (i.e. finding the non-zero Nash Equilibrium: for instance, see Nash, 1950; Nash, 1951; Dutta, 1999; Vasin \& Morozov, 2005);

- formalization of the governing/stimulating body objective in the social network and finding conditions for the characteristics of subjects and types of activity, which fulfillment leads to function objective of the governing/stimulating body reaching its maximum.

Remark 1. Let us emphasize that in this work we reject the frequently used (sometimes even considered as well-established) assumption about the same significance/value of subjects within a localized group of a social network (i.e., within an interest group, where the opinions of different subjects are quite close), and, in some works, from a mathematical point of view, this assumption is present in disguise, although the description text of these works speaks about the difference in the significance levels of the subjects. In our opinion, this assumption is not strictly scientifically substantiated, and although it facilitates the construction and, most importantly, the study of mathematical models having a focus on various problems of social networks, it substantially narrows down the limit of their adequacy and, therefore, reduces the reliability of the 
obtained results, which later on are used for putting forward hypotheses, making forecasts, formulating recommendations, etc. For example, let us consider a social network $S$ with $n$ subjects, (i.e., $\operatorname{card}(S N)=n$ ), where the $i$-th subject is characterized by numerical indicators/parameters $x_{i} \geq 0$ and $w_{i} \in(0,1)$, meaning the chosen action (strategy; response; behavior; evaluation; contribution; donation; etc.) and the significance/weight of this subject, respectively (generally speaking, the number of indicators - numeric and non-numeric, characterizing each subject of a social network, can be much greater, but at the moment we are interested in only two numeric indicators - the chosen action and the significance of the subject). It is obvious that even if for some event, for example, the United States presidential election, the cost of the $i$-th subject's chosen action dominates over the cost of the $j$-th subject's action, $x_{i}>x_{j}>0$, then it is quite possible that the real (true; effective; final) value $w_{i} \cdot x_{i}$ of the $i$-th subject's action will be less than the real value $w_{j} \cdot x_{j}$ of the $j$-th subject's action, $0<w_{i} \cdot x_{i}<w_{j} \cdot x_{j}$. This circumstance is typical not only for social networks, it takes place very often in various areas of our daily life - in educational processes, in financial and economic assessments, in determining the quality of the environment, in sports competitions, etc.: for example, the knowledge of a student or a pupil in a particular discipline may be assessed differently in different educational establishments with different weights (here the weight will denote the quality of education). In the third section of this paper, we will get back to this question, exploring it in more details. End of Remark (EOR)

\section{Mathematical Models, and their Description and Interpretation}

Let us consider a social network $S$ with subjects $\left\{s_{i}\right\}_{i=1, n}$ of a combined nature (see Introduction), and let us suppose that the subject $s_{i}$ is characterized by three indicators $\left(w_{i} ; x_{i} ; c_{i}\right)$, where the numerical indicator $w_{i}>0$ characterizes the significance/weight of the subject (within this section, without loss of generality, we will assume that the weights $\left\{s_{i}\right\}_{i=1, \overline{1, n}}$ of network subjects are normalized, i.e. $\left.w_{i} \in(0,1), \forall i=\overline{1, n} ; \sum_{i=1}^{n} w_{i}=1\right)$; a numerical indicator $\quad x_{i} \geq 0$ characterizes the cost of an action (often instead of an "action cost", simply the term "action" is used), chosen by the subject independently or under the social network or off-network informational influence of other subjects, and/or the governing/stimulating body, and/or off-network circumstances regarding any 
information events (see Remark 1); a numerical indicator $c_{i} \in(0,1)$, where $\sum_{i=1}^{n} c_{i}>2$, characterizes the type of network activity (for example, awareness/propaganda; organization/mobilization; action/reaction; etc., for instance, see Vegh, 2003), within which the subject chooses his action. It is important to note that sometimes non-numerical data are used as activity types, for example, they are associated with different colours (for instance, see Zhilyakova, 2019).

Since the real value of the $s_{i}$ subject action is inversely proportional to the type of network activity, within which this subject chooses his action (the greater is the type of activity, the less is the real value of the action: $\frac{w_{i} \cdot x_{i}}{c_{i}}, \forall i=\overline{1, n}$ ), then the $s_{i}$ subject's applied effort to joint action related to some information event in the network will be successful only when the difference between the joint efforts of the network subjects and the above proportion exceeds the given necessary threshold $L_{S}>0$, i.e. if a $\sum_{j=1}^{n} w_{j} \cdot x_{j}-\frac{w_{i} \cdot x_{i}}{c_{i}}>L_{s}$. Therefore, the subject $s_{i}$ must choose such an action $x_{i}$, so that his contribution to the overall success is maximal, i.e. we have

$$
f_{s_{i}}(w, c ; x) \stackrel{\text { def }}{\equiv} w_{i} \cdot x_{i} \cdot\left(\langle w, x\rangle-\frac{w_{i} \cdot x_{i}}{c_{i}}-L_{s}\right) \rightarrow \max _{w, x},
$$

where $w=\left(w_{1}, w_{2}, \ldots, w_{n}\right), \quad c=\left(c_{1}, c_{2}, \ldots, c_{n}\right), \quad x=\left(x_{1}, x_{2}, \ldots, x_{n}\right)^{T},\langle\bullet \cdot \bullet\rangle$ means an inner product of vectors.

Suppose that the weights $w$ of the social network's $S$ subjects are known (or are given a priori, or found based on some reason, for example, following the approach proposed in the next section). Then, taking into account the fact that the function $f_{s_{i}}(w, c ; x)$ depends on all variables $x_{1}, x_{2}, \ldots, x_{n}$, is the objective function of only the $s_{i}$-th subject of the network, then for all actions $x$ of the subjects $\left\{s_{i}\right\}_{i=1, \bar{n}}$ of the social network $S$ we obtain the problem of vector unconditional optimization

$$
F_{S}(w, c ; x) \stackrel{\text { def }}{\equiv}\left\{f_{s_{1}}(w, c ; x), f_{s_{2}}(w, c ; x), \ldots, f_{s_{n}}(w, c ; x)\right\} \rightarrow \max _{w, x}
$$


which using the Goal attainment method of Gembicki (for instance, see Gembicki, 1973; Gembicki \& Haimes, 1973; Steuer, 1986; Fleming \& Pashkevich, 1986; Aleksejevs, Guseinovs, Medvedev, \& Guseynov, 2017) is reduced to the following one-criterion conditional optimization problem:

$$
\left\{\begin{array}{l}
\bar{F}_{S}(w, c ; \bar{x}) \stackrel{\text { def }}{\equiv} x_{n+1} \rightarrow \max _{\bar{x}} \\
f_{s_{i}}(w, c ; x)-x_{n+1} \cdot f_{s_{i}}^{*} \geq f_{s_{i}}^{*}, i=\overline{1, n}
\end{array}\right.
$$

where $\bar{X}=\left(x_{1}, x_{2}, \ldots, x_{n}, x_{n+1}\right)^{T}, \quad f_{s_{i}}^{*}$ is the solution to the one-criterion scalar unconstrained optimization problem (1) for the given weights $w$ of the network subjects, i.e. $f_{s_{i}}^{*}=\arg \max _{x} f_{s_{i}}(w, c ; x)$. It is possible to find in an explicit form the values $\left\{f_{s_{i}}^{*}\right\}_{\forall i=\overline{1, n}}$, using the necessary condition (point $x$ is a critical point, i.e. the inequalities $\frac{\partial f_{s_{i}}(w, c ; x)}{\partial x_{i}}=0, i=\overline{1, n}$ should be satisfied) and the sufficient condition (the Hessian matrix $H f_{s_{i}}$, where $\left(H f_{s_{i}}\right)_{j, k}=\left\{\frac{\partial^{2} f_{s_{i}}(w, c ; x)}{\partial x_{j} \partial x_{k}}\right\}_{j, k}$, should be a negative-definite matrix) for the maximum of the function $f_{s_{i}}(w, c ; x)$ to exist (as we assumed that the weights $w$ are known, then function $f_{s_{i}}(w, c ; x)$ is a function of the $x$ argument) in $x_{i} \geq 0, i=\overline{1, n}$.

Indeed, from the condition $\frac{\partial f_{s_{i}}(w, c ; x)}{\partial x_{i}}=0, i=\overline{1, n}$ we obtain that the nonzero stationary point $x$ of the $f_{s_{i}}(w, c ; x), i=\overline{1, n}$ functions has coordinates

$$
x_{i}=\frac{\left(1-L_{S}\right) \cdot \sum_{j=1}^{n} \frac{c_{j}}{2-c_{j}}+L_{S}}{\sum_{j=1}^{n} \frac{c_{j}}{2-c_{j}}-1} \cdot \frac{c_{i}}{w_{i} \cdot\left(2-c_{i}\right)}, i=\overline{1, n},
$$

in which the fulfillment of the obvious necessary inequality $\sum_{j=1}^{n} \frac{c_{j}}{2-c_{j}}>1$ is automatically ensured by the condition $\sum_{i=1}^{n} c_{i}>2$, that we have imposed above 
on the types of activity $c=\left(c_{1}, c_{2}, \ldots, c_{n}\right)$. Further, taking into account the fact that

$$
\frac{\partial^{2} f_{s_{i}}(w, c ; x)}{\partial x_{j} \partial x_{k}}=\left\{\begin{array}{l}
\underbrace{2 \cdot w_{i}^{2}}_{>0} \cdot \underbrace{\left(1-\frac{1}{c_{i}}\right)}_{\begin{array}{c}
<0 \\
c_{i}
\end{array} \text { otherwise }}<0 \text { if } i=j=k,
\end{array}\right.
$$

then we can assert that the stationary point $x$ with coordinates (4) is the maximum point for the function $f_{s_{i}}(w, c ; x)$ from (1), i.e. point $x$ with coordinates (4) is a non-zero equilibrium action of network subjects $\left\{s_{i}\right\}_{i=1, n}$ of network $S$. Finally, substituting (3) in the expression of the function $f_{s_{i}}(w, c ; x)$ from (1), we can find the sought values $\left\{f_{s_{i}}^{*}\right\}_{\forall i=\overline{1, n}}$ :

$$
\begin{gathered}
f_{s_{i}}^{*}=-\frac{\left(1-L_{S}\right) \cdot \sum_{j=1}^{n} \frac{c_{j}}{2-c_{j}}+L_{S}}{\sum_{j=1}^{n} \frac{c_{j}}{2-c_{j}}-1} \cdot \frac{c_{j}}{2-c_{j}} \times \\
\times\left(\frac{\left(1-L_{S}\right) \cdot \sum_{j=1}^{n} \frac{c_{j}}{2-c_{j}}+L_{S}}{\sum_{j=1}^{n} \frac{c_{j}}{2-c_{j}}-1} \cdot\left(\sum_{j=1}^{n} \frac{c_{j}}{2-c_{j}}-\frac{1}{2-c_{i}}\right)-L_{S}\right) .
\end{gathered}
$$

There remains to consider the case of inactivity (that is, the perfectly admissible case of zero actions) of network subjects. From (5) and

$$
\begin{gathered}
\left.f_{s_{i}}(w, c ; x)\right|_{\left(x /\left\{x_{i}\right\}\right)=0}=\left(1-\frac{1}{c_{i}}\right) \cdot w_{i}^{2} \cdot x_{i}^{2}-L_{S} \cdot w_{i} \cdot x_{i}, \\
\left.\frac{\partial f_{s_{i}}(w, c ; x)}{\partial x_{i}}\right|_{\left(x /\left\{x_{i}\right\}\right)=0}=2 \cdot \underbrace{\left(1-\frac{1}{c_{i}}\right)}_{<0} \cdot \underbrace{w_{i}^{2} \cdot x_{i}}_{>0}-\underbrace{L_{S} \cdot w_{i}}_{>0}<0
\end{gathered}
$$

follows that null point $x=0$ also is an equilibrium point by Nash. An interesting statement was proved in (Fedyanin \& Chkhartishvili, 2010) that if $F_{S}(w, c ; x)$ has a Nash equilibrium point and if the actions of at least one of the network 
subjects are equal to zero, then this equilibrium point will be exactly the null point $x=0$.

So, the solution to the one-criterion conditional optimization problem (4), where the significance/value $\left\{w_{i}\right\}_{i=\overline{1, n}}$ of subjects $\left\{s_{i}\right\}_{i=\overline{1, n}}$ of a network $S$ are assumed to be known a priori, and $\left\{f_{s_{i}}^{*}\right\}_{\forall i=\overline{1, n}}$ are found according to formula (6), determines the Pareto optimal choice of actions of network subjects: the value of any of the criteria in the vector unconstrained optimization problem (2) can be improved only by deterioration of the values of the remaining criteria. Here, without going into details, we would like to note that the problem (3) and the problem

$$
\sum_{i=1}^{n} f_{s_{i}}(w, c ; x)=\langle w, x\rangle \cdot\left(\langle w, x\rangle-L_{s}\right)-\sum_{i=1}^{n} \frac{w_{i}^{2} \cdot x_{i}^{2}}{c_{i}} \rightarrow \max _{x}
$$

are not equivalent problems, although both of these one-criterion problems are generated by the same original problem (2) of vector unconditional optimization, and are different ways to solve (2) by reducing it to a one-criterion problem: we insist that the problem (3) is more preferable because of its scientifically based derivation from (2) (see: Coello Coello, Lamont, \& van Veldhuizen, 2007; Steuer, 1986; Keeney \& Raiffa, 1993; Gembicki, 1973).

Now let us discuss three of the many possible objectives of a network control/governing body (for instance, see Gubanov et al., 2010; Volodenkov, 2015; McKenney \& White, 2018; Goyal, Karamchandani, Chatterjee, \& Manjunath, 2019): (A) the objective of neutralizing the actions of network subjects; (B) the objective of a softly regulated weakening of the actions of network subjects; $(\mathrm{C})$ the purpose of a softly regulated strengthening of the actions of network subjects.

In the case where the objective of the network control/governing body is (A), then we can formulate the optimization problem

$$
G_{(A)}(w, c ; x) \stackrel{\text { def }}{\equiv} \lambda \cdot\left\langle w, \arg \max _{\bar{x}} \bar{F}_{S}(w, c ; \bar{x})\right\rangle \rightarrow \max _{c},
$$

where $\lambda \leq 0$ is a parameter, the value of which is set by the control/governing body. For example, the choice $\lambda=-1$ in (7) corresponds to the fact that the objective of the control/governing body is to create such types $c=\left(c_{1}, c_{2}, \ldots, c_{n}\right)$ of network activity so that the Pareto-optimal solution $x=\arg \max _{\bar{x}} \bar{F}_{S}(w, c ; \bar{x})$ of the network subjects would turn into a benefit in the amount $-x_{n+1}$, thus, turned out to be as much damaging as the amount total actions made by network subjects. The choice of $\lambda=0$ in (7) corresponds to the fact that the objective of the control/governing body is to exclude non-zero Nash equilibrium actions of 
network subjects. Obviously, in order to achieve that, the control/governing body only needs to succeed in creation of network activity types $c=\left(c_{1}, c_{2}, \ldots, c_{n}\right)$ that will satisfy inequality $\sum_{i=1}^{n} c_{i}<2$ or $c_{\max } \leq \frac{2}{n+1}$, where $c_{\max }=\max _{i=1, n} c_{i}$ (in (Fedyanin \& Chkhartishvili, 2010) a more accurate/better estimate has been found).

In the case where the objective of the control/governing body in the network is (B), then we can formulate the optimization problem

$$
\left\{\begin{array}{l}
G_{(B)}(y) \stackrel{\text { def }}{\equiv} \sum_{i=1}^{n} w_{i} \cdot \alpha_{i} \cdot y_{i}^{-\beta_{i}} \rightarrow \min _{y}, \\
\prod_{i=1}^{n} y_{i}^{\gamma_{i}}=Y, \\
w_{i}>0, y_{i}>0, \alpha_{i}>0, \beta_{i}>0, \gamma_{i}>0, \forall i=\overline{1, n} ; Y>0,
\end{array}\right.
$$

where $\left\{w_{i}\right\}_{i=1, n}$ are the values/weights of network subjects (without breaking the generality, we can assume that $\left.w_{i} \in(0,1), \forall i=\overline{1, n} ; \sum_{i=1}^{n} w_{i}=1\right) ;\left\{y_{i}\right\}_{i=1, n}$ are the sought-for costs of efforts/influences of the control/governing body; $Y$ is the maximum total cost of effort that the control/governing body can afford (often $Y$ is referred to as a resource); $\left\{\alpha_{i}\right\}_{i=\overline{1, n}},\left\{\beta_{i}\right\}_{i=1, n},\left\{\gamma_{i}\right\}_{i=1, n}$ are controllable parameters of the control/governing body: $\alpha_{i}$ characterizes the initial type of network activity (i.e., before the action of the governing/stimulating body), where the subject $s_{i} \in S$ choses his action; $\beta_{i}$ characterizes the degree of weakening of the influence (i.e., the opposite response to the applied effort) of the control/governing body on the subject $s_{i} \in S ; \gamma_{i}$ characterizes the degree of resource use when the effort $y_{i}$ is applied.

It is not difficult to verify that the optimization problem (8) has the following global solution: 


$$
\begin{aligned}
& y^{\min }=\left\{y_{i}^{\min }\right\}_{i=1, n}=\left\{\left(\frac{Y}{\prod_{j=1}^{n}\left(\frac{w_{j} \cdot \alpha_{j} \cdot \beta_{j}}{\gamma_{j}}\right)^{\frac{\gamma_{j}}{\beta_{j}}}}\right)^{\frac{1}{\beta_{i} \cdot \sum_{j=1}^{n} \frac{\gamma_{j}}{\beta_{j}}}} \cdot\left(\frac{w_{i} \cdot \alpha_{i} \cdot \beta_{i}}{\gamma_{i}}\right)^{\frac{1}{\beta_{i}}}\right\}_{i=\overline{1, n}}, \\
& G_{(B)}^{\min }=G_{(B)}\left(y^{\min }\right)=\left(\frac{\prod_{j=1}^{n}\left(\frac{w_{j} \cdot \alpha_{j} \cdot \beta_{j}}{\gamma_{j}}\right)^{\frac{\gamma_{j}}{\beta_{j}}}}{Y}\right)^{\frac{1}{\sum_{j=1}^{n} \frac{\gamma_{j}}{\beta_{j}}}} \cdot \sum_{i=1}^{n} \frac{\gamma_{i}}{\beta_{i}} .
\end{aligned}
$$

Let us note that if we assume $\gamma_{i}=1, \forall i=\overline{1, n}$ and introduce new designations $z_{i}=\ln y_{i}, \theta_{i}=\frac{1}{\beta_{i}}$, then the essential constraint of problem (8) takes the form $\sum_{i=1}^{n} z_{i}=Y$, and the objective function $G_{(B)}(y)$ takes the form $G_{(B)}(y)=\sum_{i=1}^{n} w_{i} \cdot \alpha_{i} \cdot e_{i}^{-\frac{z_{i}}{\theta_{i}}}$, which coincides with the objective function derived in (Fedyanin \& Chkhartishvili, 2010) from four assumptions/requirements: dependence $g(y)$ of the activity type $c_{i}$ of the subject $s_{i} \in S$ from the impact of the control/governing body must be (a) continuous; (b) asymptotically tend to zero with increasing influence of control/governing body; (c) equal to the type of subject at zero level before impact; (d) decrease monotonically. The mathematical formalization of (a), (b) and (d) requirements in the language of differential equations means that the dependence of the type of the subject's activity on the impact of the control/governing body is described by the equation $g_{i}^{\prime}(y)=-\delta_{i} \cdot g_{i}(y)$, and requirement (c) means the condition $\left.g_{i}(y)\right|_{y=0+0}=\alpha_{i}$. The solution of the obtained boundary value problem will be a function $g_{i}(y)=\alpha_{i} \cdot e^{-\delta_{i} \cdot y_{i}}$, and, therefore, we have obtained an objective function 
$\sum_{i=1}^{n} g_{i}(y)$, which coincides with the objective function of the problem (8) when denoting $\delta_{i}=\frac{1}{\theta_{i}}$.

In the case when the objective of the control/governing body in the network is $(\mathrm{C})$, then the optimization problem can be formulated as:

$$
\left\{\begin{array}{l}
G_{(C)}(y) \stackrel{\text { def }}{\equiv} \prod_{i=1}^{n} y_{i}^{\beta_{i}} \rightarrow \max _{y}, \\
\sum_{i=1}^{n} \mu_{i} \cdot y_{i}^{\gamma_{i}}=Y, \\
y_{i}>0, \beta_{i}>0, \mu_{i}>0, \gamma_{i}>0, \forall i=\overline{1, n} ; Y>0,
\end{array}\right.
$$

where $\beta_{i}$ characterizes the degree of strengthening of the influence (i.e., direct response to the applied effort) of the control/governing body on the subject $s_{i} \in S ; \mu_{i}$ characterizes the weight/effectiveness of the resource used when the effort $y_{i}$ is applied; the rest of parameters have the same meaning as in the problem (8).

It is not difficult to verify that the optimization problem (9) has the following global solution:

$$
\begin{gathered}
y^{\max }=\left\{y_{i}^{\max }>0\right\}_{i=1, n}=\left\{\left(\frac{Y \cdot \beta_{i}}{\mu_{i} \cdot \gamma_{i} \cdot \sum_{j=1}^{n} \frac{\beta_{j}}{\gamma_{j}}}\right)^{\frac{1}{\gamma_{i}}}\right\}_{i=1, n}, \\
G_{(C)}^{\max }=G_{(C)}\left(y^{\max }\right)=\sum_{i=1}^{n}\left(\frac{Y \cdot \beta_{i}}{\mu_{i} \cdot \gamma_{i} \cdot \sum_{j=1}^{n} \frac{\beta_{j}}{\gamma_{j}}}\right)^{\gamma_{i}} .
\end{gathered}
$$

\section{Determining Importance Rates of Subjects in Social Networks}

Let us consider again a social network $S$ with subjects $\left\{s_{i}\right\}_{i=\overline{1, n}}$ of a combined nature, and again let us suppose that the subject $s_{i}$ is characterized by 
three indicators $\left(w_{i} ; x_{i} ; c_{i}\right)$, which has the same meaning as in the previous section of this work. Let us suppose that during a period of time $T=\left\{t_{1}, t_{2}, \ldots, t_{K}\right\}$ (as a time unit in $T$ one can pick, for example, day, week, month, etc.), $M$ significant events were discussed in the social network $S$, and the subject $s_{i} \in S$ has actively participated in $M_{i}$ of them, $M_{i} \leq M, i=\overline{1, n}$. Let us denote by $m$ the number of those unique different events $M$, where all subjects $\left\{s_{i}\right\}_{i=\overline{1, n}}$ have actively participated. Let us denote by $x_{i, k, j}$ the measured integral cost/assessment/indicator for the various activities of the subject $s_{i} \in S \quad(i=\overline{1, n})$ within the $j$-th $(j=\overline{1, \mathrm{~m}})$ event within a $t_{k}$ time unit. Then the following expression can be interpreted as the effectiveness/success of the "network activity" of the subject $s_{i} \in S$ during a time unit $t_{k}$ within the frames of all $m$ events:

$$
E_{i, k}=A \cdot \sum_{j=1}^{m} x_{i, k, j} \cdot \xi_{j}+B, \forall(i=\overline{1, n}, k=1, K),
$$

where $\xi_{j}$ is the desired weight/significance of the integral assessment of the $s_{i} \in S$ subject's activities within the $j$-th event for the $t_{k}$ time unit; the constants $A, B$ are assumed to be known and are found from Fisher statistic.

Remark 2. In fact, in (10) the weights $\xi$ also depend on the indices $i=\overline{1, n}$, and $k=\overline{1, K}$, i.e., in general case $\xi=\left\{\xi_{i, k, j}\right\}_{i=1, \overline{1, n} ; j=\overline{1, m}}^{i \overline{1}}$. Taking in formula (10) $\xi=\left\{\xi_{j}\right\}_{j=\overline{1, m}}$, we have assumed on default that each event has its own rock-solid importance, which is constant for different subjects of the network for the entire period of time $T=\left\{t_{1}, t_{2}, \ldots, t_{K}\right\}$. Obviously, this is not the case: the same event, even for the same person at different times, can be of completely different value and importance. There could be given countless examples from the field of science and technology, from economics, medicine, politics, sociology, etc. EOR

Expression (10) is a system of linear algebraic equations with $n \cdot K$ equations and $n \cdot K+m$ unknowns (due to the unknown $\left\{\xi_{j}\right\}_{j=1, m}$, the left side $\left\{E_{i, k}\right\}_{i=1, \bar{n} ; k=\overline{1, K}}$ of the system is also unknown), and, therefore, system (10) is not a normal system, and its solution should be refined (Tikhonov \& Arsenin, 1977 ). However, before proceeding to the construction of an algorithm for solving 
system (10), let us ask ourselves the question, even if $\left\{\xi_{j}\right\}_{j=1, m}$ are found, how to determine the weights/importance $\left\{w_{i}\right\}_{i=1, n}$ of the network subjects? In order to answer this question, let us return to Remark 2, where it has been stated that in general case $\xi=\left\{\xi_{i, k, j}\right\}_{i=\overline{1, n} ; j=\overline{1, m}}^{i, \overline{1}}$. So, if $\left\{\xi_{i, k, j}\right\}_{i=\overline{1, n}, j=\overline{1, m}}^{i, \overline{1},}$ were found, then for each fixed index $i \in\{1,2, \ldots, n\}$ they would average $\left\{\xi_{i, k, j}\right\}_{i=\overline{1, n} ; j=\overline{1, m}}^{i \overline{1, K}}$ over the indices $j=\overline{1, m}, k=1, K$, and the obtained result would be taken as (arithmetic mean; root mean square; cubic mean; etc.) weight/significance $w_{i}, i=\overline{1, n}$. Another way is to find the norm of matrices (for example, Euclidean norms; $L_{p, q}$ norm, etc.) $\left\{\xi_{i, k, j}\right\}_{i=1, \overline{1, n} ; j=\overline{1, m}}^{i, \overline{1}}$ for each fixed index $i \in\{1,2, \ldots, n\}$. These methods of determining weights $\left\{w_{i}\right\}_{i=1, n}$ are quite logical and legitimate. In our case, we have $\left\{\xi_{j}\right\}_{j=\overline{1, m}}$, and the above-mentioned methods (also other legitimate methods, for instance, see Feller, 1968; Bellman, 1997) do not allow determining the values of the $n$ parameters by indirectly measured $m$ numbers. The approach outlined below, which is based on the fundamental concept of Tikhonov's regularization (Tikhonov \& Arsenin, 1977), allows, first, to reduce system (10) to a normal system with $n \cdot K+m$ equations and unknowns, and second, to find a stable pseudosolution (regularized solution) of the resulting system, and, thirdly, to determine the desired $\left\{w_{i}\right\}_{i=1, n}$ by the numbers $n \cdot K+m$ that are already found.

Let us introduce an extended matrix $\tilde{X}$ having the size of $(n \cdot K) \times(n \cdot K+m)$, whose elements $\tilde{x}_{i, k, j}$ are calculated by the formula

$$
\tilde{x}_{i, k, j} \stackrel{\text { def }}{\equiv}\left\{\begin{array}{ccc}
-1, & \text { if }(j>m) \wedge(K \cdot i+k=j+(K-m)) ; \\
0, & \text { if }(j>m) \wedge(K \cdot i+k \neq j+(K-m)) ; \\
A \cdot x_{i, k, j}, & \text { if } \quad j \leq m .
\end{array}\right.
$$

Then system (10) takes the form

$$
\sum_{j=1}^{n \cdot K+m} \tilde{x}_{i, k, j} \cdot \tilde{\xi}_{j}=-B, i=\overline{1, n}, k=\overline{1, K},
$$

where

$$
\tilde{\xi}_{j}{ }_{\text {def }} \equiv\left\{\begin{array}{l}
\xi_{j} \text { if } \quad j \leq m ; \\
E_{i, k} \text { if } \quad m<j: \forall i=\overline{1, n}, \forall k=\overline{1, K} j=m+(i-1) \cdot K+k
\end{array}\right.
$$


System (12) using the designation $\left.\Psi \stackrel{\text { def }}{\equiv}\left\{\tilde{\xi}_{j}\right\}_{j=\overline{1, n \cdot K+m}}, \quad \tilde{B} \equiv(\underbrace{\operatorname{def}}_{n \cdot n})^{-B, \ldots,-B}\right)^{T}$ takes the matrix form

$$
\tilde{X} \tilde{\Psi}=\tilde{B} .
$$

So, the original system (10) is reduced to system (13) with $n \cdot K$ equations and $n \cdot K+m$ unknowns $\tilde{\Psi}=\left\{\tilde{\xi}_{j}\right\}_{j=1, n \cdot K+m}$. It is obvious that system (13) is an underdetermined system. Following the fundamental monograph (Tikhonov \& Arsenin, 1977), a pseudo-solution of the underdetermined system (13) there will be called the vector

$$
\tilde{\Psi}^{*}=\arg \min _{\tilde{\Psi}}\|\tilde{X} \tilde{\Psi}-\tilde{B}\|,
$$

where by $\|\cdot\|$ we denote Schur norm, $\|$ vector $\|_{2} \leq \sqrt{\sum_{k} v e c t o r_{k}^{2}}$, $\|$ matrix $\|_{2,1} \leq \sqrt{\sum_{i} \sum_{j} \text { matrix }_{i j}^{2}}$.

Obviously, to find a pseudosolution of system (13), according to (14), it is required to solve an unconditional extremal problem $\|\tilde{X} \tilde{\Psi}-\tilde{B}\| \rightarrow \min _{\tilde{\Psi}}$, for the existence of a solution it is required to fulfill a condition of the first variation of the functional $V(\tilde{\Psi}) \stackrel{\text { def }}{\equiv}\|\tilde{X} \tilde{\Psi}-\tilde{B}\|$, i.e. we have the equation

$$
0=\delta V(\tilde{\Psi})=V(\tilde{\Psi}+\Delta \tilde{\Psi})-V(\tilde{\Psi})=2 \cdot\left\langle\Delta \tilde{\Psi}, \tilde{X}^{T} \tilde{X} \tilde{\Psi}+\frac{1}{2} \cdot \tilde{X}^{T} \tilde{X} \Delta \tilde{\Psi}-\tilde{X}^{T} \tilde{B}\right\rangle,
$$

from which, due to arbitrariness of increment $\Delta \tilde{\Psi}$, follows the system

$$
\tilde{X}^{T} \tilde{X} \tilde{\Psi}=\tilde{X}^{T} \tilde{B}
$$

which, unlike system (13), is a normal system with $n \cdot K+m$ equations and the same number of unknowns $\left\{\tilde{\xi}_{j}\right\}_{j=1, \overline{1, K+m}}$.

So, we got that the pseudosolution $\tilde{\Psi}^{*}$ of the underdetermined system (13) is the classical solution of the normal system (15), and vice versa. However, the main matrix $\tilde{X}^{T} \tilde{X}$ of system (15) may turn out to be an ill-conditioned matrix, and, therefore, we cannot solve it by any direct methods (analytical or numerical 
methods). In other words, the solution $\tilde{\Psi}$ to the normal system (15) cannot be expressed by a formula $\tilde{\Psi}=\left(\tilde{X}^{T} \tilde{X}\right)^{-1} \tilde{X}^{T} \tilde{B}$, from which, by virtue of the Cauchy inequality $\|\tilde{\Psi}\| \leq\left\|\left(\tilde{X}^{T} \tilde{X}\right)^{-1}\right\| \cdot\left\|\tilde{X}^{T} \tilde{B}\right\|$, the stability of the pseudo-solution $\tilde{\Psi}^{*}$ of system (13) would follow.

Remark 3. It should be noted that $\operatorname{det}\left(\tilde{X}^{T} \tilde{X}\right) \ll 1$, generally speaking, is not a criterion for the ill-conditioned matrix $\tilde{X}^{T} \tilde{X}$. Therefore, in order to clarify the question of ill-conditioned of a particular matrix, it is necessary to calculate not its determinant in order to reveal how small it is in comparison with one, but the conditionality number

$$
\operatorname{cond}\left(\tilde{X}^{T} \tilde{X}\right)=\operatorname{cond}\left(\left(\tilde{X}^{T} \tilde{X}\right)^{-1}\right)=\left\|\tilde{X}^{T} \tilde{X}\right\| \cdot\left\|\left(\tilde{X}^{T} \tilde{X}\right)^{-1}\right\|=\frac{\lambda_{\max }\left(\tilde{X}^{T} \tilde{X}\right)}{\lambda_{\min }\left(\tilde{X}^{T} \tilde{X}\right)} \geq 1
$$

where $\lambda_{\min }\left(\tilde{X}^{T} \tilde{X}\right)$ and $\lambda_{\max }\left(\tilde{X}^{T} \tilde{X}\right)$ are the minimum and maximum eigenvalues of matrix $\tilde{X}^{T} \tilde{X}$.

Further, in addition to the aforementioned problem of ill-conditioned of the normal system (15), hence, the instability of its classical solution, violation of the condition $\operatorname{det}\left(\tilde{X}^{T} \tilde{X}\right) \neq 0$ also violates the Hadamard Well-posedness of system (13) in the sense of a pseudo-solution, namely, a pseudo-solution as a solution of a normal system (15), may turn out to be non-unique, and then it is determined up to elements from the kernel ker $\tilde{X}$ of the principal matrix $\tilde{X}$. In other words, if the condition $\operatorname{det}\left(\tilde{X}^{T} \tilde{X}\right) \neq 0$ is violated, then the pseudosolution is non-unique and all of them are determined up to solutions of a homogeneous system of equations $\tilde{X} \tilde{\Psi}=0$ (this follows from the Fredholm alternative for first kind operator equations). Therefore, in the case under consideration, if $\tilde{\Psi}^{(1)}$ is some pseudosolution of system (13), then any column vector $\tilde{\Psi}=\tilde{\Psi}^{(1)}+\tilde{\Psi}^{(2)}$, where $\tilde{\Psi}^{(2)} \in \operatorname{ker} \tilde{X}$, will also be a pseudosolution of system (13), and all these solutions can differ arbitrarily from each other. Therefore, a criterion for selection of a solution must be formulated. This criterion is given by the concept of a normal pseudo-solution: a pseudo-solution with a minimal Schur norm is called a normal pseudo-solution (Tikhonov \& Arsenin, 1977). EOR

So, let us return to the normal system (15). The following iterative algorithm that is described below, which is Tikhonov's regularizing algorithm, allows us to find a normal pseudosolution of system (15). 
Step 1. A decreasing sequence of numerical parameters $\left\{\alpha_{m}\right\}_{m \in \mathbb{N}}$ is taken, for example, $\alpha_{m}=2^{-m}, m \in \mathbb{N}$, and for its two neighboring terms, for example, the first two, $\alpha=\alpha_{1}$ and $\alpha=\alpha_{2}$, by some direct method (for example, Gaussian elimination method) the following system of equations with respect to $(n \cdot K+m)^{2}$ unknowns $\left\{r_{m, i}^{\alpha}\right\}_{i, m=1, n \cdot K+m}$ is solved:

$$
\alpha \cdot r_{m, i}^{\alpha}+\sum_{k=1}^{n \cdot K+m} r_{m, k}^{\alpha} \cdot \beta_{k, i}=\left(\tilde{X}^{T} \tilde{X}\right)_{i, m}, \quad i, m=\overline{1, n \cdot K+m}
$$

where

$$
\beta_{k, i}=\sum_{p=1}^{n \cdot K+m}\left(\tilde{X}^{T} \tilde{X}\right)_{k, p} \cdot\left(\tilde{X}^{T} \tilde{X}\right)_{i, p}
$$

Step 2. For each of the parameters $\alpha=\alpha_{m_{0}}$ and $\alpha=\alpha_{m_{0}+1}$, the coordinates $\left\{\tilde{\xi}_{m}^{\alpha}\right\}_{m=1, n \cdot K+m}$ of the column vector $\tilde{\Psi}^{\alpha}$ are found using the formula

$$
\tilde{\xi}_{m}^{\alpha}=\sum_{i=1}^{n \cdot K+m} r_{m, j}^{\alpha} \cdot\left(\tilde{X}^{T} \tilde{B}\right)_{i}, \forall m=\overline{1, n \cdot K+m}
$$

Step 3. It is verified whether the condition $\left\|\tilde{\Psi}^{\alpha_{m_{0}}}-\tilde{\Psi}^{\alpha_{m_{0}+1}}\right\| \leq \varepsilon$, is satisfied where $\varepsilon$ is a desired reasonable accuracy (for example, $\varepsilon=10^{-3}$ ) of the approximate normal pseudo-solution to the exact/theoretical solution (which is unknown). If the answer is positive, then at the end of the algorithm an approximate normal pseudosolution $\tilde{\Psi}^{* \alpha}=\left\{\tilde{\xi}^{\alpha}\right\}_{j=1, n \cdot K+m}$ is found, and, therefore, the sought ones $\left\{E_{i, k}\right\}_{i=1, \bar{n} ; k=1, K}$ and $\left\{\xi_{j}\right\}_{i=1, m}$ are found in the original system (10):

$$
\begin{gathered}
E_{i, k}=\tilde{\xi}_{j}^{\alpha} \text { at } j=\overline{m+1, n \cdot K+m} ; \\
\xi_{j}=\tilde{\xi}_{j}^{\alpha} \text { at } j=\overline{1, m} .
\end{gathered}
$$

If $\left\|\tilde{\Psi}^{\alpha_{m_{0}}}-\tilde{\Psi}^{\alpha_{m_{0}+1}}\right\|>\varepsilon$, then the transition to Step 2 is carried out, letting $\alpha_{m_{0}}=\alpha_{m_{0}+1}$ and $\alpha_{m_{0}+1}=\alpha_{m_{0}+2}$. The theoretical justification, which is presented in (Dmitriev \& Guseynov, 1995), guarantees the finiteness of this algorithm and finding of an approximate normal pseudosolution of system (10).

Finally, the formula 
SOCIETY. INTEGRATION. EDUCATION

Proceedings of the International Scientific Conference. Volume III, May 28 ${ }^{\text {th }}-29^{\text {th }}$, 2021. 254-272

$$
w_{i}=\frac{\left\|E_{i}\right\|_{2}^{2}}{\|E\|_{2,1}^{2}}, \forall i=\overline{1, n},
$$

where $E_{i}=\left\{E_{i, k}\right\}_{k=1, \bar{K}}, E=\left\{E_{i}\right\}_{i=1, \bar{n}}$, determines the desired weights/significance $\left\{w_{i}\right\}_{i=1, n}$ of network subjects $\left\{s_{i}\right\}_{i=1, n}$ in the social network $S$.

\section{Conclusions}

This work studies social networks, the subjects of which are of a combined nature - they can behave both actively and passively. Under some additional assumptions, relationships are established between the selected actions of the subjects, the types of network activity, the coefficients of the significance of the subjects, and the efforts of the control/governing body in the network. The established relations allow formulating a vector unconstrained optimization problem for finding coordinated optimal actions of network subjects. Further, using the Goal attainment method of Gembicki, the formulated vector optimization problem is reduced to a one-criterion conditional optimization problem. In addition, in this work, three single-criterion problems of conditional optimization are formulated for the optimal impact of the control/governing body on the types of actions of the subjects: the objective of the control/governing body in the first problem is to neutralize the actions of network subjects, in the second problem - realize softly controlled weakening of the actions of network subjects, in the third problem is to implement softly regulated strengthening of the actions of network subjects. Also, this paper investigates the issue of finding the significance coefficients of network subjects for a certain set of measured integral indicators of network subjects: a mathematical model is constructed and studied, a regularizing iterative algorithm is proposed for solving the constructed model, one of the possible mechanisms for using the found normal pseudo-solution of the model for determination of the significance coefficients of the network subjects is proposed.

\section{References}

Aleksejevs, R., Guseinovs, R., Medvedev, A.N., \& Guseynov, Sh.E. (2017). On a Multicriterion Problem of Planning Maritime Cargo Transportation. Journal of Traffic and Transportation Engineering, 4, 124-146.

Amati, V., Lomi, A., \& Mira, A. (2018). Social Network Modeling. Annual Review of Statistics and Its Application, 5(1), 343-369.

Bellman, R. (1997). Introduction to Matrix Analysis. Philadelphia: SIAM Publishing. 
Bukharin, S.N. \& Malkov, S.Yu. (2010). On the Question of Mathematical Modelling of Information Interactions. Information Warfares, 2(14), 14-20.

Bukharin, S.N., Kovalev, V.I., \& Malkov, S.Yu. (2009). On Formalization of Information Field Concept. Information Warfares, 4(12), 2-9.

Coello Coello, C.A., Lamont, G.B., \& van Veldhuizen, D.A. (2007). Evolutionary Algorithms for Solving Multi-Objective Problems. New York: Springer-Verlag.

Chernets, V., Bazlova, T., \& Ivanova, E. (2010). Influence Through Social Networks. Moscow: FOKUS-MEDIA.

Chkhartishvili, A.G., Gubanov, D.A., and Novikov, D.A. (2019). Social Networks: Models of Information Influence, Control and Confrontation. Cham, Switzerland: Springer Nature.

Dmitriev, V.I. \& Guseynov, Sh.E. (1995). On matching the resolving power and detailedness of inverse problems solution. Herald of the Moscow State University, Series 15: Computational Mathematics and Cybernetics, 1, 24-27.

Dutta, P.K. (1999). Strategies and Games: Theory and Practice. Boston: MIT Press.

Farris, P., Pfeifer, Ph.E., \& Johnson, R.R. (2009). The Value of Networks. Charlottesville: Darden School of Business, University of Virginia, Darden Case No. UVA-M-0645.

Fedyanin, D.N. \& Chkhartishvili, A.G. (2010). On a problem of information control in social networks. Large-Scale Systems Control, 31, 265-275.

Feller, W. (1968). Introduction to Probability Theory and its Applications, Vol I. New York: John Wiley \& Sons.

Fleming, P.J., \& Pashkevich, A.P. (1986). Application of Multiobjective Optimization to Compensator Design for SISO Control Systems. Electronics Letters, 22(5), 258-259.

Fowler, J.H. \& Christakis, N.A. (2011). Connected: The Surprising Power of Our Social Networks and How They Shape Our Lives. New York: Little, Brown and Company.

Galam, S. (2012). Sociophysics: A Physicist's Modeling of Psycho-political Phenomena. New York: Springer-Verlag.

Gembicki, F.W. (1973). Vector Optimization for Control with Performance and Parameter Sensitivity Indices. Ph.D. Thesis, Department of System Engineering. Cleveland: Case Western Reserve University.

Gembicki, F.W., \& Haimes, Y.Y. (1975). Approach to Performance and Sensitivity Multiobjective Optimization: The Goal Attainment Method. IEEE Transactions on Automatic Control, 29(6), 769-771.

Goyal, M., Karamchandani, N., Chatterjee, D., \& Manjunath, D. (2019). Maintaining Ferment: On Opinion Control over Social Networks. Proceedings of IEEE 58th Conference on Decision and Control, 5217-5222.

Grabich, N. \& Rusinowska, A. (2010). A Model of Influence in a Social Network. Theory and Decision, 69(1), 69-96.

Gubanov, D.A. (2020). Influence in Social Networks: Formalization Variants. Large-Scale Systems Control, 85, 51-71.

Gubanov, D.A., Novikov D.A., \& Chkhartishvili, A.G. (2009). Social Media Influence Models. Control in Socio-Economic Systems, 7, 205-281.

Gubanov, D.A., Novikov D.A., \& Chkhartishvili, A.G. (2010). Social Networks: Models of Information Influences, Control and Confrontations. Moscow: FizMatLit Publishing.

Hoede, C. \& Bakker, R. (1982). A Theory of Decisional Power. Journal of Mathematical Sociology, 8, 309-322.

Jackson, M. (2008). Social and Economic Networks. Princeton: Princeton University. 
SOCIETY. INTEGRATION. EDUCATION

Proceedings of the International Scientific Conference. Volume III, May $28^{\text {th }}-29^{\text {th }}$, 2021. 254-272

Johnson, N., Turnbull, B., Maher, Th., \& Reisslein, M. (2021). Semantically Modeling Cyber Influence Campaigns (CICs): Ontology Model and Case Studies. IEEE Access, 9, 93659382.

Keeney, R.L. \& Raiffa, H. (1993). Decisions with Multiple Objectives: Preferences and Value Trade. Cambridge: Cambridge University Press.

Kovarik, B. (2015). Revolutions in Communication: Media History from Gutenberg to the Digital Age. New York: Bloomsbury Academic Publisher.

Malkov, S.Yu. (2016). Social Organization and Historical Process: Possibilities of Mathematical Modelling. Moscow: LIBROKOM Publishing.

McKenney, D., \& White, T. (2018). Towards Distribution-based Control of Social Networks. Computational Social Networks, 5, Article ID: 1236.

Nash, J. (1950). Equilibrium Points in n-person Games. Proceedings of the National Academy of Sciences, 36(1), 48-49.

Nash, J. (1951). Non-Cooperative Games. The Annals of Mathematics, 54(2), 286-295.

Nguyen, V.X., Xiao, G., Xu, X.-J., Wu, Q., \& Xia, Ch.-Y. (2020). Dynamics of Opinion Formation under Majority Rules on Complex Social Networks. Scientific Reports, 10, Article ID: 456, 9 p.

Razis, G., Anagnostopoulos, I., \& Zeadally, Sh. (2020). Modeling Influence with Semantics in Social Networks: a Survey. ACM Computing Surveys, 53(1), Article ID: 7, 61.

Reed, D.P. (1999). That Sneaky Exponential: Beyond Metcalfe's Law to the Power of Community Building. Retrieved from https://www.deepplum.com/ dpr/locus/gfn/reedslaw.html

Reed, D.P. (2001). The Law of the Pack. Harvard Business Review, 79(2), 23-4, 154. PMID: 11213694.

Rusinowska, A. \& Swart, H. (2007). Generalizing and Modifying the Hoede-Bakker Index. Theory and Applications of Relational Structures as Knowledge Instruments. Notes in Artificial Intelligence, 2, 60-88.

Simeonov, S. (2006). Metcalfe's Law: More Misunderstood than Wrong? HighContrast: Innovation \& venture capital in the post-broadband era. Retrieved from https://blog.simeonov.com/2006/07/26/metcalfes-law-more-misunderstood-than-wrong/

Steuer, R.E. (1986). Multiple Criteria Optimization: Theory, Computation, and Application. New York: John Wiley \& Sons.

Tikhonov, A.N. \& Arsenin, V.Ya. (1977). Solutions of Ill-Posed Problems. New York: V.H.Winston \& Sons Publishing.

Vasin, A.A. \& Morozov, V.V. (2005). Game Theory and Models of Mathematical Economics. Moscow: Lomonosov Moscow State University Press.

Vegh, S. (2003). Classifying Forms of Online Activism: The Case of Cyberprotests Against the World Bank. In McCaughey, M., \& Ayers, M.D. (Eds.), Cyberactivism: Online Activism in Theory and Practice, (72-73). New York: Routledge \& CRC Press.

Volodenkov, S.V. (2015). Internet-Communications in the Global Space of Modern Political Governance. Moscow: Moscow State University Press.

Xiong, F. \& Liu, Y. (2014). Opinion formation on social media: An empirical approach. Interdisciplinary Journal of Nonlinear Science, 24(1), Article ID: 013130, 8 p.

Zhilyakova, L.Yu. (2019). Modeling the Structure of MIMO-Agents and Their Interactions. In Kuznetsov, S., \& Panov, A. (Eds.), Artifical Intelligence, (3-16), 1093. Cham: Springer. 